\title{
Against the "Ordinary Summing" Test for Convergence
}

\author{
G. C. GoDDu University of Richmond
}

\begin{abstract}
One popular test for distinguishing linked and convergent argument structures is Robert Yanal's Ordinary Summing Test. Douglas Walton, in his comprehensive survey of possible candidates for the linked/ convergent distinction, advocates a particular version of Yanal's test. In a recent article, Alexander Tyaglo proposes to generalize and verify Yanal's algorithm for convergent arguments, the basis for Yanal's Ordinary Summing Test. In this paper I will argue that Yanal's ordinary summing equation does not demarcate convergence and so his Ordinary Summing Test fails. Hence, despite Walton's recommendation or Tyaglo's generalization, the Ordinary Summing Test should not be used for distinguishing linked argument structures from convergent argument structures.
\end{abstract}

\begin{abstract}
Résumé: L’Épreuve "Ordinary Summing" de Robert Yanal est une façon courante de distinguer des prémisses dépendantes des prémisses indépendantes. Dans son exposé détaillé des différentes discussions sur la distinction entre ces prémisses, Douglas Walton plaide en faveur d'une version de l'épreuve de Yanal. Dans un article récent, Alexander Tyaglo propose de généraliser et de vérifier la méthode algorithmique de Yanal, qui est à la base de son épreuve "Ordinary Summing". Dans cet article j'avance que sa méthode ne distingue pas les prémisses dépendantes des prémisses indépendantes, et donc son épreuve échoue. Par conséquent, malgré la recommendation de Walton ou la généralisation de Tyaglo, on ne devrait pas employer l'épreuve "Ordinary Summing" pour différencier ces prémisses.
\end{abstract}

Keywords: linked, convergent, ordinary sum, conditional probability

\section{Introduction}

Many, though certainly not all, introductory logic textbooks devote some attention to argument structure and the diagramming of argument structure. In particular, significant focus is placed on distinguishing linked argument structure from convergent argument structure. The intuition guiding the distinction is that in some arguments some of the premises link together to form a single reason for the conclusion, while in others the premises each constitute separate reasons which converge on the conclusion. If the intuition grounding the distinction is sound, then the general problem is, for any set of premises for a single conclusion, to partition the set of premises into reasons for the conclusion. Some reasons might be constituted by multiple premises, while others by a single premise. Unfortunately, beyond the basic intuition, the nature of the linked/convergent distinction is highly contentious and a quick perusal of introductory logic textbooks will gener- 
ate numerous competing and incompatible tests for partitioning the premise set and so for determining the exact linked/convergent structure of a given argument.

One test of particular interest is Robert Yanal's Ordinary Summing Test (Yanal, 1988,1991, 2003). In Douglas Walton's comprehensive survey of possible candidates for the linked/convergent distinction, he concludes that a particular version of Yanal's proposed test, while not without shortcomings, is the best (Walton, 1996, 181). In a recent article, (Tyaglo, 2003) Alexander Tyaglo proposes to generalize and verify Yanal's algorithm for convergent arguments, the basis for Yanal's Ordinary Summing Test. Even the most significant criticism of Yanal's proposal, that of David Conway, merely claims that while Yanal's test "captures as well as possible any intuitive sense behind the notion of convergent arguments" it "doesn't have the importance it seemed to promise" (Conway, 1991, 154). Conway does not suggest that Yanal's test is incorrect, but merely that if Yanal's test is correct it does not seem that the linked/convergent distinction is very useful.

In this paper I will argue for a claim stronger than Conway's. I will argue that Yanal's ordinary summing equation does not demarcate convergence and so his Ordinary Summing Test fails. Hence, despite Walton's recommendation or Tyaglo's generalization, the Ordinary Summing Test should not be used for distinguishing linked argument structures from convergent argument structures. In Section 2, I shall present and clarify Yanal's ordinary summing equation and his Test for making the linked/convergent distinction. In Section 3, I shall present generalized versions of Yanal's ordinary summing equation, discuss Tyaglo's "verification" of these equations, and present some difficulties for using these generalized equations as the basis for a Generalized Ordinary Summing Test, i.e., a test that applies to arguments with any finite number of premises, rather than just arguments with two premises. In Section 4, I shall argue that the ordinary summing equation fails to demarcate convergence and so Yanal's Test, and any generalization of it, fails to successfully distinguish linked and convergent argument structures. In Section 5, I shall (a) consider various attempts to avoid the problems of Section 4 and (b) argue that no option succeeds. I shall conclude that despite any intuitive appeal the test may acquire from success in some cases, the test fails in equally significant cases and so is to be abandoned.

\section{Yanal's Ordinary Summing Equation}

Consider the following examples:

(A) (1) She's either in the study or in the kitchen.

(2) She's not in the study.

(3) She's in the kitchen. (Yanal, 2003)

and 
(B) (1) She typically goes to the kitchen around this time to make a cup of tea. (2) I just saw her walking in the general direction of the kitchen.

(3) She's in the kitchen. (Yanal, 2003).

According to Yanal, "It is just such cases as A and B that lead us to think there is a real distinction to be drawn" (Yanal 1991, p. 137).'

In other words, these examples are meant to be canonical examples of linked versus convergent argument structures - examples that show that at least in some cases we can intuitively group premises into reasons; i.e., we can recognize, independently of any test, that some premises link together to form a single reason (as in A), whereas others are separate reasons in themselves (as in B). Given that these examples are supposed to make us accept that there is a real distinction to be drawn, any test of convergence that fails to demarcate the canonical examples correctly, must be incorrect. I shall argue, in Section 4, that this is exactly what the Ordinary Summing Test does-it fails to demarcate the canonical examples correctly. But first I turn to stating and explicating the Ordinary Summing Test for convergence.

According to Yanal, "The probability of the conclusion of an argument with independent premises is the ordinary sum of the probability of each premise. The probability of the conclusion of an argument with dependent premises is not the ordinary sum of the probability of each premise" (Yanal, 1991, 140). "The probability of conclusions from arguments whose premises are dependent jumps beyond the ordinary sum of these premises" (140). Other than a slight terminological change, Yanal's recent formulation is effectively the same:

Convergent arguments have premises whose probabilities sum in the ordinary way. Linked arguments have premises whose probabilities don't sum in the ordinary way. Linked arguments have reasons that "jump" ordinary probability sums $(2003,3-4)$.

But exactly what probabilities are being summed and what is it for them to sum "ordinarily"?

Consider first Yanal's descriptions of what he calls Ordinary Summing. Concerning a non-specific example, $P 1, P 2$, therefore $C$. He writes:

Suppose $\mathrm{P} 1$ in itself lends 0.3 probability to $\mathrm{C}$. Suppose $\mathrm{P} 2$ in itself lends 0.4 probability to $\mathrm{C}$.... We can think of the ordinary sum of the probabilities this way. The probability of $\mathrm{C}$ given $\mathrm{P} 1$ is 0.3 . Take 0.3 from 1.0 leaving 0.7 . Call 0.7 "what is unknown." When $\mathrm{P} 2$ is brought in, we know 0.4 of 0.7 , or $0.4 \times 0.7$, which is 0.28 more than we knew before. In sum we know the conclusion $\mathrm{C}$ with 0.58 probability $(0.3+0.28)(1991,140)$.

Concerning argument (B), Yanal writes:

Suppose that 4 out of 5 days she goes to the kitchen at this time to make tea. Then that premise confers on its conclusion a probability of 0.8 . Now there is a remainder uncertainty of 0.2 of whether she's in the kitchen now. The second premise, that I've seen her walking in the direction of the kitchen, 


\section{G. C. Goddu}

removes some of that uncertainty. Suppose that I'm generally right about what I see-say $75 \%$ of the time. Then we can say that the second premise reduces the uncertainty by $75 \%$, or 0.75 times 0.2 , which is $0.15, \ldots$. We're then entitled to add the probabilities of the two premises, $0.8+0.15$, and see that our two premises support the conclusion with a probability of 0.95 . We'll call this the ordinary way of summing probabilities $(2003,3)$.

So what probabilities are being summed in the case of Ordinary Summing? In Yanal's examples it is the probability that each premise "lends to" or "confers on" the conclusion. Hence, despite the fact that Yanal describes his tests for linked/ convergent arguments in terms of "premises whose probabilities sum in the ordinary way", it is not the probabilities of the premises that are being summed, but rather the conditional probabilities of the conclusion given each premise. After all, it is our uncertainty concerning the conclusion that is being removed by each premise.

So what exactly is the ordinary sum of the relevant conditional probabilities? Suppose you have two premises $\mathrm{P} 1$ and $\mathrm{P} 2$ and a conclusion $\mathrm{C}$. Let the probability of $\mathrm{C}$ given $\mathrm{P} 1$, i.e., $\operatorname{pr}(\mathrm{C} \mid \mathrm{P} 1)$, be $p r$, and the probability of $\mathrm{C}$ given $\mathrm{P} 2$, i.e., $\operatorname{pr}(\mathrm{C} \mid \mathrm{P} 2)$, be $p r_{2}$. Designate $o s_{t, 2}$ the ordinary sum of two conditional probabilities, $p r_{1}$ and $p r_{2}$. In Yanal's manner of speaking the ordinary sum of two conditional probabilities is just (a) the degree of uncertainty relative to $C$ removed by $P 1$ added to (b) the degree of uncertainty relative to $\mathrm{C}$ removed by $\mathrm{P} 2$ from whatever uncertainty is left over from that removed by $\mathrm{P} 1$. In algebraic terms:

$$
o s_{1,2}=p r_{1}+\left(1-p r_{1}\right) p r_{2}
$$

Put another way, $o s_{1,2}=\left(p r_{1}+p r_{2}\right)-p r_{1} p r_{2}$; i.e., add the percentage of cases in which P1 makes $\mathrm{C}$ true to the percentage of cases in which P2 makes $\mathrm{C}$ true and subtract once the percentage of cases in which both $\mathrm{P} 1$ and $\mathrm{P} 2$ make $\mathrm{C}$ true, for otherwise those cases are being double counted.

What is the relevance of the ordinary sum? Let the probability of $\mathrm{C}$ given both $\mathrm{P} 1$ and $\mathrm{P} 2$; i.e., $\operatorname{pr}(\mathrm{C} \mid \mathrm{P} 1 \& \mathrm{P} 2)$, be $p r_{1,2}$. In very specific circumstances, $p r_{1,2}$ is equal to $o s_{1,2}$. In other words, the conditional probability of the conclusion given both premises is equal to the ordinary sum of the conditional probabilities of the conclusion given each premise separately.

Accordingly, if the probability of the conclusion given the premises is the ordinary sum of the relevant conditional probabilities, then Yanal says the argument is convergent. If the probability of the conclusion given the premises "jumps beyond" the ordinary sum, then the argument is linked. For now let us assume that by "jumps beyond" Yanal merely means "is greater than" (though this assumption will be examined in greater detail in Section 4 below). In this case, we have the following test for distinguishing linked and convergent structures, at least for twopremise arguments: 
The Ordinary Summing Test: For any argument with exactly two premises, the argument is convergent iff $p r_{l, 2}=o s_{1,2}$, and the argument is linked iff $\mathrm{pr}_{1,2}>o s_{l, 2}$.

Hence, given the test, if we discover that $p r_{l, 2}=o s_{l, 2}$, then the two premises are each separate reasons for the conclusion, but if $\mathrm{pr}_{1,2}>o s_{1,2}$ then the argument consists of a single reason for the conclusion and this single reason is constituted by both premises together.

\section{On Generalizing the Ordinary Summing Test}

As currently stated, the Ordinary Summing Test is applicable only to two premise arguments. Can the Test be generalized to apply to arguments with more than two premises? Presumably, the first thing we require is a generalized ordinary summing formula - a formula for determining the ordinary sum in cases requiring summing more than two conditional probabilities. Following Yanal's talk of eliminating uncertainty, generalizing the ordinary summing formula is straightforward. Suppose we have three premises, $\mathrm{P} 1, \mathrm{P} 2$, and $\mathrm{P} 3$ and a conclusion $\mathrm{C}$ such that $\mathrm{pr}$, $=0.6, p r_{2}=0.5$, and $p r_{3}=0.7$. What then is the ordinary sum of these three conditional probabilities, i.e., what is $o s_{1,2,3}$ ? Speaking with Yanal, P1 removes 0.6 of the uncertainty; of the remaining 0.4 uncertainty $\mathrm{P} 2$ removes $1 / 2$, or. 0.2 ; of the remaining 0.2 uncertainty $P 3$ removes $7 / 10$ or 0.14 , leaving only an uncertainty of 0.06 . Hence, the ordinary sum of the three conditional probabilities is $0.6+0.2+$ 0.14 , or 0.94 .

Put as an equation, the formula for three premises is:

$$
o s_{1,2,3}=p r_{1}+\left(1-p r_{1}\right) p r_{2}+\left(1-p r_{1}\right)\left(1-p r_{2}\right) p r_{3} \text {. }
$$

Generalizing further, the equation for $n>2$ premises is,

$$
o s_{l, 2, \ldots, n}=p r_{1}+\left(1-p r_{1}\right) p r_{2}+\ldots+\left(1-p r_{1}\right)\left(1-p r_{2}\right) \ldots\left(1-p r_{n-1}\right) p r_{n} \text {. }
$$

In other words, no matter how many premises, start with the conditional probability of the first premise; add to that the amount of uncertainty that the second premise removes from the uncertainty left by the first premise; add to that the amount of uncertainty that the third premise removes from what is left by the first and second premises; keep repeating the process until all premises have been accounted for.

Alternatively, given that $o s_{1,2}=p r_{1}+\left(1-p r_{1}\right) p r_{2}$ and that $p r_{1}+\left(1-p r_{1}\right) p r_{2}$ is part of the equation for $o s_{I, 2,3}$, we can define the ordinary sum for arguments with three or more premises recursively as follows:

$$
o s_{l, 2,3}=o s_{l, 2}+\left(1-o s_{l, 2}\right) p r_{3}
$$


and

$o s_{l, 2, \ldots, n}=o s_{l, 2 \ldots, n-1}+\left(1-o s_{l, 2, \ldots, n-l}\right) p r_{n}$.

Ignoring different, though in my view unfortunate ${ }^{2}$, notational choices, it is these recursively defined generalizations of Yanal's original equation that, along with the original equation, Alexander Tyaglo claims "to verify" by showing that they satisfy four "natural essential conditions" (Tyaglo, 2003, 65).

Tyaglo's "natural" and "essential" conditions are as follows:

C1: For any set of premises, the ordinary sum lies between 0 and 1 .

C2: For any set of premises, the ordinary sum will be the same regardless of the order in which the relevant conditional probabilities are summed.

C3: For any set of premises such that the probability of $\mathrm{C}$ given some premise in the set is 1 , the ordinary sum will be 1 .

C4: For any set of premises such that the probability of $C$ given one of those premises is 0 , the ordinary sum of the entire set will be the same as the ordinary sum without that premise (Tyaglo, 2003, 65).

Though Tyaglo does not offer a rigorous proof, he correctly claims that the ordinary summing equations satisfy these conditions. Indeed, intuitively the ordinary summing equations should satisfy these conditions. For example, given that $(i)$ no conditional probability is less than 0 and greater than 1 , (ii) the initial uncertainty can be at most 1, and (iii) each premise is removing, at most, some of whatever uncertainty remains, the ordinary sum must be between 0 and 1 , and so intuitively the ordinary summing equations satisfy $\mathrm{C} 1 . \mathrm{C} 2$ just mandates that the order of the removing of uncertainty is irrelevant to the final result. In addition, any premise that is sufficient for the conclusion and so confers a probability of 1 on the conclusion will remove all remaining uncertainty. As a result, as $\mathrm{C} 3$ requires, the ordinary sum will be 1 and none of the remaining premises will alter the ordinary sum for there is no uncertainty left for them to remove. Finally, since any premise that confers 0 probability on the conclusion removes none of the uncertainty, it will have no bearing on the ordinary sum, as $\mathrm{C} 4$ stipulates.

Suppose we grant, even without rigorous proof, that the ordinary summing equations satisfy Tyaglo's four conditions. In what sense though, if any, should we grant that satisfying these conditions "verifies" the ordinary summing equations, or more significantly, the Ordinary Summing Test? At most we should grant that satisfying these conditions shows that the ordinary summing equations meet some basic requirements for a coherent probability calculus; i.e., the probabilities must all be between 0 and 1 , and so since we are summing, order does not matter; once we get to 1 we can go no higher, and adding 0 does not alter the sum. But while failing to satisfy the basic requirements of a coherent probability calculus would indeed be a problem, satisfying these requirements does not thereby show that the ordinary summing equations are adequate for their proposed function of underpinning a test for distinguishing convergent from linked argument struc- 
tures. Indeed, the relevant "verification" we require is that ordinary summing is in fact a correct indicator of convergent argument structures, and nothing Tyaglo offers shows that. Hence, for all the "verification" Tyaglo has given, it could turn out that the ordinary summing equations satisfy the basic requirements of a coherent probability calculus, but fail to accurately demarcate convergent argument structures.

In Section 4 below, I will argue that not only could it turn out this way, but that it in fact does turn out that even though the ordinary summing equations satisfy Tyaglo's "natural" and "essential" conditions, they do not satisfy the most essential condition of accurately demarcating convergence. First, however, I shall demonstrate that even with the generalized ordinary summing equations in hand, articulating a satisfactory Generalized Ordinary Summing Test is not a simple or obvious generalization of the Ordinary Summing Test. Indeed, the case of just three premises poses significant questions concerning exactly what ordinary summing demarcates.

Given the generalized ordinary summing equations, how might we go about generalizing the Ordinary Summing Test? One natural proposal replaces $p r_{t, 2}$ with $p r_{l, \ldots, n}$ and $o s_{l, 2}$ with $o s_{l \ldots, n}$ The resulting test is,

Generalized Ordinary Summing Test 1: For any argument with $n$ premises, the argument is convergent iff $p r_{1 \ldots, n}=o s_{l, \ldots, n}$; the argument is linked iff $\mathrm{pr}_{l, \ldots, n}>$ os ${ }_{1, \ldots, n}$.

According to Generalized Test 1 , if we discover that the actual conditional probability of the conclusion given all the premises is equal to the ordinary sum of the conditional probabilities of the conclusion given each premise individually, then the argument must be convergent. If the actual conditional probability is greater than the ordinary sum, then the argument must be linked. Unfortunately, this natural proposal can easily be shown to be inadequate.

When the number of premises, $n$, equals two, there are exactly two ways of partitioning the premises into reasons for a conclusion. Letting $\{$,$\} group premises$ into distinct reasons, the two possible patterns are:

(i) $\{\mathrm{P} 1, \mathrm{P} 2\}$

and

(ii) $\{\mathrm{P} 1\}$ and $\{\mathrm{P} 2\}$.

In other words, either the two premises link to form a single reason or each premise is a reason in its own right. When $n=2$, the Generalized Ordinary Summing Test just is the Ordinary Summing Test, so if $\mathrm{pr}_{1.2}=0 s_{1,2}$, then the correct grouping of premises is the second and the argument structure is convergent, but if $p r_{1,2}>0 s_{1,2}$, then the correct grouping of premises is the former and the premises are linked. So far so good.

But when $n=3$ there are, assuming premises cannot be part of more than one reason, five possible patterns, viz.: 
(i) $\{\mathrm{P} 1, \mathrm{P} 2, \mathrm{P} 3\}$

(ii) $\{\mathrm{P} 1\}$ and $\{\mathrm{P} 2, \mathrm{P} 3\}$

(iii) $\{\mathrm{P} 2\}$ and $\{\mathrm{P} 1, \mathrm{P} 3\}$

(iv) $\{\mathrm{P} 1, \mathrm{P} 2\}$ and $\{\mathrm{P} 3\}$

(v) $\{\mathrm{P} 1\}$ and $\{\mathrm{P} 2\}$ and $\{\mathrm{P} 3\}$.

Pattern $(i)$ we might say represents a pure linked structure, for all the premises are linked to form a single reason, whereas pattern $(v)$ represents a pure convergent structure since each premise is its own individual reason. Patterns (ii)-(iv), on the other hand, we might say all represent impure linked and convergent structures, or hybrid structures; i.e., structures where (a) some, but not all of the premises link into single reasons, and (b) there are at least two reasons that converge on the conclusion. In other words, hybrid structures involve both premise linkage and premise or reason convergence. ${ }^{3}$

Right away there is a problem. Generalized Test 1 only demarcates two sorts of structures, viz., convergent and linked. Yet for any $n>2$, there will be at least three kinds of structures; viz., purely convergent, purely linked, and hybrid. Hence, Test 1 cannot by itself be used to determine for any argument structure whether it is purely convergent, purely linked, or hybrid. At the very least then, Test 1 requires refining in order to be an adequate demarcation test for arguments with three or more premises.

How might such a refinement proceed? Given the alleged significance of ordinary summing, determining which, if any, of the five possible patterns are indicated when $\mathrm{pr}_{1,2,3}=o s_{1,2,3}$ seems an appropriate starting point. Since ordinary summing is supposed to correspond with convergence, there are three reasonable possibilities for what $p r_{l, 2,3}$ equaling $o s_{l, 2,3}$ demarcates: (a) pure convergence alone, i.e., pattern (v) only; (b) impure convergence alone, i.e., one of patterns (ii)-(iv) only; ${ }^{4}$ or (c) convergence, either pure or impure, i.e., one of patterns (ii) $-(v)$.

Based on our intuitions about how ordinary summing works, especially the fact that the order in which we sum the conditional probabilities does not matter, one might suspect that ordinary summing demarcates pure convergence. If the order of ordinary summing does not matter, then the premises must, seemingly, be all independent of each other such that none of the premises are linked with any of the others, and so ordinary summing must, seemingly, demarcate pure convergence. Indeed, in most cases, if $p r_{1,2,3}=o s_{l, 2,3}$ the pattern cannot be hybrid, and, assuming it is not purely linked, must therefore be purely convergent. In particular, it can be shown that if no single premise is alone sufficient for the conclusion, then if $p r_{1,2,3}=o s_{1,2,3}$, the pattern cannot be hybrid. [For a full proof of this claim, see Appendix One]. Unfortunately, it is not universally true that if $p r_{1,2,3}=o s_{1,2,3}$ the pattern cannot be hybrid.

To see this, suppose that $p r_{1}=1$. Hence, given that one of the premises is alone sufficient for the conclusion, according to $\mathrm{C} 3$, no other premises have any bearing 
on the ordinary sum and $o s_{1,2,3}=1$. But if $p r_{1}=1$, then the actual probability of the conclusion given all the premises, viz., $p r_{1,2,3}$ must also be 1. Hence, $p r_{1,2,3}=o s_{1,2,3}$. But now consider $\mathrm{P} 2$ and $\mathrm{P} 3$ and assume that at least the Ordinary Summing Test is adequate. Either $p r_{2,3}=o s_{2,3}$, or it doesn't. If it does, then P2 and P3 must be convergent, in which case, given that the whole argument is convergent, the pattern must be the purely convergent one or $(v)$. If not, then, supposing $\mathrm{pr}_{2,3}>\mathrm{O}_{2,3}$, $\mathrm{P} 2$ and $\mathrm{P} 3$ are linked and so the pattern must be the hybrid pattern (ii). Hence, if one of the premises is alone sufficient for the conclusion, then $p r_{1,2,3}$ equaling $o s_{1,2,3}$ cannot distinguish whether the argument is an example of pattern $(v)$ or one of (ii)-(iv).

Suppose, based on the fact that if all we know is that $p r_{t, 2,3}=o s_{t, 2,3}$ then the argument could be either purely convergent or hybrid, we refine Generalized Test 1 as follows:

Generalized Ordinary Summing Test 2: For any argument with $n$ premises, the argument is purely convergent or hybrid iff $\mathrm{pr}_{t, \ldots, n}=o s_{1, \ldots, n}$, and the argument is purely linked iff $p r_{1, \ldots, n}>o s_{1, \ldots, n^{*}}$

Test 2 , however, fares no better than Test 1 , for both biconditionals of Test 2 are demonstrably false.

To see this consider the following two arguments:

(C) (1) Either she is in the kitchen, the study or the bedroom.

(2) She is not in the study.

(3) She is not in the bedroom.

(4) She is in the kitchen.

and

(D) (1) Either she is in the kitchen, the study, or the bedroom.

(2) She is neither in the study nor the bedroom.

(3) She typically goes to the kitchen around this time to make a cup of tea.

(4) She is in the kitchen.

Intuitively, (C) has a purely linked structure, whereas (D) has a hybrid structure in which $\mathrm{P} 1$ and $\mathrm{P} 2$ are linked, but $\mathrm{P} 3$ is its own separate reason. Both arguments are deductively valid so $\mathrm{pr}_{1,2,3}=1$. In neither argument is a single premise sufficient for the conclusion, so in both cases $o s_{1,2,3}<1$. [See Appendix Two.] Hence, in both cases $p r_{t, 2,3}>o s_{t, 2,3}$. Since (D) is hybrid, but $p r_{l, 2,3}>o s_{1,2,3}$, it is not true that an argument is purely linked iff $p r_{1,2,3}>o s_{1,2,3}$. At the same time, since (D) is hybrid, according to the first biconditional of Test 2, (D) ought to be such that $\mathrm{pr}_{1,2,3}=$ $o s_{1,2,3}$. But since $p r_{1,2,3}>o s_{1,2,3}$, the first biconditional of Test 2 is also false.

More generally, what the faults with Tests 1 and 2 show is that merely knowing that $p r_{t, 2,3}=o s_{1,2,3}$ or $p r_{t, 2,3}>o s_{t, 2,3}$ is insufficient for being able to demarcate any of the three possible structures. The best we might be able to do is know that if $p r_{1,2,3}>o s_{1,2,3}$, then the argument is not purely convergent and if $p r_{1,2,3}=o s_{1,2,3}$ then 
the argument is not purely linked. But merely knowing that an argument is not purely linked or not purely convergent leaves open too many possibilities regarding its actual structure. Indeed, the problem will only get worse as the number of premises increases and so the number of non-equivalent hybrid cases increases.

Regardless, perhaps the generalized ordinary summing equations can be supplemented with, or perhaps even supplanted by, further ordinary summing calculations in order to successfully demarcate each argument structure. For example, in the case of three premise arguments the following two options might seem worth pursuing.

Option 1. Compare the values of $p r_{1,2}, p r_{l, 3}$, and $p r_{2,3}$ with those of $o s_{l, 2}, o s_{l, 3}$, and $o s_{2,3}$ respectively. Hypothesize that a three premise argument is (a) purely convergent iff for all three pairs $p r_{x, y}=o s_{x, y}$; (b) hybrid iff for exactly one pair $p r_{x, y}$ $>o s_{x, y}$ (that will be the linked pair); and (c) purely linked iff for all three pairs $p r_{x, y}$ $>o s_{x, y}$.

Option 2. Determine the ordinary sums of each of the following pairs of conditional probabilities: (i) $p r_{l, 2}$ and $p r_{3}$; (ii) $p r_{l, 3}$ and $p r_{2}$; and (iii) $p r_{2,3}$ and $p r_{l}$. In effect this is treating each three-premise argument as a two-premise argument in which one premise is the conjunction of two of the original premises. Hypothesize that a three-premise argument is (a) purely convergent iff for all three pairs the ordinary sum $=p r_{1,2,3} ;$ (b) hybrid iff for exactly one pair the ordinary sum $=p r_{1,2,3}$; and (c) purely linked iff for all three pairs $p r_{1,2,3}>$ the ordinary sum.

Notice that both of the options rely heavily on the success of the Ordinary Summing Test; i.e., that the ordinary sum of a pair of conditional probabilities equaling the appropriate actual conditional probability demarcates convergence. I suspect that as the number of premises increases and so the number of nonequivalent structures requiring demarcation increases, more and more reliance will have to be placed on calculating the sums of an increasing number of pairs of conditional probabilities. Hence, given this reliance, if ordinary summing fails to demarcate convergence in the two-premise case, then no test based on ordinary summing will adequately distinguish convergent from linked structures. In the next section I intend to demonstrate that Yanal's Ordinary Summing Test fails to demarcate convergence, and so the prospects for determining argument structure based on the ordinary summing equations are bleak indeed.

To summarize the main points of this section, firstly, Alexander Tyaglo's "verification" of the generalized ordinary summing equations fails to verify the most important condition, i.e., that ordinary summing demarcates convergence. Secondly, while generalizing Yanal's original ordinary summing equation is relatively straightforward, adequately generalizing the test for convergence based on the ordinary summing equations is not. Thirdly, merely utilizing the appropriate ordinary summing equation based on how many premises an argument involves will not be sufficient for determining the argument's structure. Most likely, further appeals to the ordinary sums of pairs of conditional probabilities will be required. 
Hence, knowing whether Yanal's original ordinary summing equation demarcates convergence is essential for determining the prospects of any Generalized Ordinary Summing Test. I turn, then, to a critical examination of Yanal's basic Ordinary Summing Test.

\section{Incorrect Classification of Arguments}

My strategy in this section will be to argue that the Ordinary Summing Test classifies argument (B) incorrectly. Since (B), viz:

(1) She typically goes to the kitchen around this time to make a cup of tea.

(2) I just saw her walking in the general direction of the kitchen.

(3) She's in the kitchen

is supposed to be obviously or pre-theoretically convergent, if the Test classifies (B) incorrectly, then whatever property ordinary summing demarcates, it is not convergence.

Consider first the following argument:

(E) (1) Al shot at the target.

(2) Bob shot at the target.

(3) Therefore the target was hit.

Suppose that the probability that the target was hit given that Al shot at it is 0.9 and the probability that it was hit given that Bob shot at it was 0.8 . So in 0.9 of the cases $\mathrm{Al}$ hits the target and in $0.8 \mathrm{Bob}$ hits the target. Assuming that Al's hitting the target is stochastically independent of Bob's hitting the target, in 0.9 multiplied by 0.8 of the cases, or 0.72 , they both hit the target. Hence, the ordinary sum probability that the target was hit is, $0.9+0.8-0.72$, or $1.7-0.72$, or 0.98 . Assuming that the conclusion is equivalent to "the target was hit by $\mathrm{Al}$ or the target was hit by Bob", then the actual support $\mathrm{P} 1$ and $\mathrm{P} 2$ provide to $\mathrm{C}$ in this case is indeed 0.98 . Hence, according to Yanal's Ordinary Summing Test (E) is convergent. I suspect this result matches our intuitions, for (E), like (B), appears to be a canonical example of a convergent argument.

But now consider another apparently canonical convergent argument:

(F) (1) The mail was delivered today.

(2) Tom went to work.

(3) It is a weekday.

Suppose the mail is delivered on all days but Sunday, regardless of holidays. Hence, the probability of the conclusion given the first premise is $5 / 6$ or 0.83 . Suppose Tom ultimately works five out of seven days, but $30 \%$ of his days off are weekdays and $70 \%$ weekends. In addition, given that Tom is off, no weekday is more probable than any other and neither day of the weekend is more probable than the other. In this case P2 makes it 0.88 probable that it is a weekday. To see this, 
consider 100 weeks or 700 days. Tom goes to work 500 days. Of the 200 days he does not go to work, $70 \%$ or 140 , are weekend days and either Saturday or Sunday is equally likely. Hence, he does not go to work 70 Saturdays and 70 Sundays. Hence, he goes to work on 30 Saturdays and 30 Sundays. Of the 200 days he does not go to work, $30 \%$ or 60 are weekdays, and each weekday is equally likely. Hence, he does not go to work on 12 Mondays, 12 Tuesdays, etc. Hence, he does go to work 88 days of each weekday. Hence, given that he goes to work, the probability that it is a weekday is $440 / 500$ or 0.88 .

The ordinary sum of 0.83 and 0.88 is $0.83+(1-0.83) 0.88$ or $0.83+(0.17) 0.88$ or $0.83+0.1496$ or approximately 0.98 . The actual conditional probability is the percentage of cases in which it is a weekday given that both the mail came and Tom went to work. The mail comes and Tom goes to work on 470 days; 440 weekdays and 30 Saturdays. Hence, the actual probability is $440 / 470$ or approximately 0.936 . Hence, the actual support is less than the ordinary sum.

According to the Ordinary Summing Test, an argument is convergent iff the actual conditional probability is equal to the ordinary sum and linked iff the actual conditional probability is greater than the ordinary sum. But despite (F)'s canonical appearance, the actual conditional probability is less than the ordinary sum. Hence, according to the Ordinary Summing Test $(F)$ is neither linked nor convergent.

But how can $(\mathrm{F})$ be neither? How can it be that the reasons $(\mathrm{F})$ provides for its conclusion are neither $(i)\{\mathrm{P} 1\}$ and $\{\mathrm{P} 2\}$ separately, nor $(i i)$ the single reason $\{\mathrm{P} 1$, $\mathrm{P} 2\}$ ? Perhaps single premises can be parts of more than one reason such that in the two premise case we need to consider not just patterns $(i)$ and (ii), but also (iii) $\{\mathrm{P} 1\}$ and $\{\mathrm{P} 1, \mathrm{P} 2\}$ and $($ iv) $\{\mathrm{P} 1, \mathrm{P} 2\}$ and $\{\mathrm{P} 2\}$. But neither new pattern succeeds either, since both require summing the actual conditional probability of the argument, viz., $p r_{l, 2}$, with $p r_{1}$ or $p r_{2}$. But $p r_{l, 2}=0.936$ and $p r_{1}$ and $p r_{2}$ are both greater than 0 . Hence the ordinary sum of $p r_{t, 2}$ and either $p r_{1}$ or $p r_{2}$ must still be greater than 0.936. In addition, both (iii) and (iv) involve a reason in which P1 and $\mathrm{P} 2$ are linked, yet since $p r_{l, 2}<o s_{l, 2}$ according to the Test, $\mathrm{P} 1$ and $\mathrm{P} 2$ are not linked. I can see no other possibilities for partitioning the premises of two premise arguments into reasons. Hence, according to the Ordinary Summing Test, the premises of $(F)$ cannot be partitioned into reasons, and $(F)$ has no reasons structure at all. But that is absurd, so the Ordinary Summing Test must be mistaken.

(E) satisfies the Test, but (F) does not, even though, intuitively, both seem convergent. Yanal's canonical example of a convergent argument, viz., (B) also seems convergent, but is it really or is it another example like (F)? Consider 100 cases in which it is her typical kitchen time. Given Yanal's proposed probability, in 80 such cases she will be in the kitchen and in 20 she will not. Now suppose in all 100 of those cases I was present to view her movements just prior to her arriving at her destination. Given that according to Yanal I am correct about what I see $75 \%$ of the time, in 60 of the 80 cases in which she is in the kitchen, I will have seen her heading for the kitchen. In 15 of the 20 of the cases in which she is not 
in kitchen I will not have seen her heading to the kitchen. In the remaining five cases in which she is not in the kitchen, I will have seen her heading for the kitchen. ${ }^{5}$ Hence, in 65 cases it is both kitchen time and I saw her heading that way, but in only 60 of them, or just under $93 \%$, will she be in the kitchen. But as Yanal correctly points out, the ordinary sum in this case is 0.95 . Hence, the actual support in (B) is not the ordinary sum, and so, contra Yanal, according to his own Ordinary Summing Test, (B) is not convergent.

Indeed, according to the Ordinary Summing Test, (B), like (F), has no reasons structure. Hence, far from being a canonical convergent argument-an argument that when compared with arguments like (A) makes us think there truly is a linked/ convergent distinction-(B) seems to be no kind of argument at all. But given the choice between rejecting the Ordinary Summing Test or affirming that the premises of (B) cannot be partitioned into reasons and that (B) has no reasons structure, I suspect most will opt for rejecting the Ordinary Summing Test, at least in its current form. After all, even knowing in the case of (B) that the actual conditional probability is less than the ordinary sum does nothing, for most people I suspect, to allay the strong pre-theoretic intuition that (B) is an exemplar convergent argument. Regardless, before examining ways in which an advocate of using ordinary sums might modify the Ordinary Summing Test, I shall briefly explore what the ordinary sum being equivalent to the actual conditional probability in fact demarcates.

When discussing the relevance of the ordinary sum in section 2 of this paper, I said that in very specific circumstances the conditional probability of the conclusion given both premises is equal to the ordinary sum of the conditional probabilities of the conclusion given each premise separately. But what exactly are those circumstances? os $s_{1,2}$ is just $p r_{1}+\left(1-p r_{1}\right) p r_{2}$, which I also pointed out is the same as $p r_{1}+p r_{2}-p r_{1} p r_{2}$. Notice, however, that this latter equation is in fact a particular case of the general disjunction rule for probabilities. Given that $\mathrm{X}$ is the probability of $\mathrm{P}$ and $\mathrm{y}$ the probability of $\mathrm{Q}$, then $\operatorname{Pr}(\mathrm{P} \vee \mathrm{Q})$, assuming $P$ and $Q$ are stochastically independent, is $(\mathrm{x}+\mathrm{y})-\mathrm{xy}^{6}$

So now reconsider (E), for which the ordinary sum equals the actual conditional probability. Firstly, the conclusion of $(E)$ is, in the circumstances, equivalent to the disjunction "Al hits the target or Bob hits the target." By the disjunction rule then, the probability of the conclusion is just the probability that $\mathrm{Al}$ hits the target added to the probability that Bob hits the target minus the probability that they both hit the target. Secondly, the probability that $\mathrm{Al}$ hits the target just is the probability that $\mathrm{Al}$ hits the target given that $\mathrm{Al}$ shoots at the target, i.e., $p r$, and likewise the probability that Bob hits the target just is the probability that Bob hits the target given that he shoots at it, i.e., $p r_{2}$. Finally, on the plausible supposition that Al's rate of success is independent of Bob's shooting at the target and Bob's rate of success is independent of Al's shooting at the target, then "Al hits the target" is stochastically independent of "Bob hits the target". Hence the probability that they both hit the 
target is just the probability that $\mathrm{Al}$ hits the target multiplied by the probability that Bob hits the target. Hence, the probability of the conclusion given the premises in the case of $(\mathrm{E})$ is $\left(p r_{1}+p r_{2}\right)-p r_{1} p r_{2}$, i.e., the ordinary sum.

In the cases of $(\mathrm{B})$ and $(\mathrm{F})$, however, neither conclusion is equivalent to a relevant disjunction, i.e., a disjunction in which the probability of each disjunct depends entirely on distinct premises in such a way that the disjuncts are stochastically independent. In addition, in the case of (E) it makes perfect sense to consider 100 cases in which both $\mathrm{Al}$ and Bob shot at the target, and to say that in 90 of those cases the target was hit because Al hit it and in 80 the target was hit because Bob hit it (and, by the way, in 72 cases the target was hit because both hit it). But in the case of (B), for example, it makes no sense to consider 100 cases in which it's both kitchen time and I saw her heading that way, and to say that in 80 of those cases she was there because it was kitchen time and in 75 because I saw her. The difference, I take it, is that in the case of $(E)$ the premises are probabilistic causes of the conclusion, whereas in (B) and (F) the premises are merely probabilistic indicators of the conclusion. Regardless, the separation of cases required for appropriately applying the disjunction rule is not possible in the case of either (B) or $(\mathrm{F})$, and so the actual probability of the conclusion given the premises is not determined by calculating the ordinary sum.

In fact, I suspect that very few arguments will satisfy the conditions required for an appropriate application of the disjunction rule and so very few arguments will satisfy the Ordinary Summing Test-certainly far fewer than most proponents of the linked/convergent distinction would traditionally classify as convergent. Given that the Ordinary Summing Test classifies so few arguments as convergent, even rejecting arguments that appear canonically convergent, and that according to the Ordinary Summing Test, many very straightforward looking arguments turn out to have no reasons structure at all, the Ordinary Summing Test, as it now stands, is to be rejected. But perhaps the Ordinary Summing Test can be modified to avoid these problematic consequences. I turn to examining some possible modifications next.

\section{Revised Ordinary Summing Tests}

Both (B) and (F) appear convergent and in both the actual support is less than the ordinary sum. Hence, neither (B) nor (F) violate what Yanal says indicates linked arguments, viz., the actual support jumps the ordinary sum. Perhaps, then, an advocate of the utility of ordinary summing can hold that in convergent arguments the actual support is equal to or less than the ordinary sum, while in linked arguments the actual support is greater than the ordinary sum and so simply revise the Ordinary Summing Test as follows:

Ordinary Summing Test A: For any argument with exactly two premises, the argument is convergent iff $\mathrm{pr}_{1,2} \# \mathrm{OS}_{l, 2}$, and the argument is linked iff $p r_{l, 2}>o s_{1,2}$. 
Test A clearly solves the problem of arguments having no structure at all, since for any argument the actual probability must be either greater than the ordinary sum or less than or equal to the ordinary sum. But, assuming that (B) and (F) are canonically convergent, then Test $\mathrm{A}$ is also problematic, for it also misclassifies arguments.

Consider (F) again, but in the following circumstances: Tom works six days out of seven instead of five out of seven; all his days off are weekend days; and he works only one Saturday in 100 . Given, then, that Tom went to work, the probability that it is a weekday must be $5 / 6$ or roughly 0.83 . At the same time, given the mail came it is still 0.83 likely that it is a weekday. In this case, the ordinary sum of (F) is $0.83+0.83(0.17)$, or 0.9711 . But given that Tom works only on one Saturday in 100 , the only days on which both Tom goes to work and the mail comes are all the weekdays and one Saturday out of 100 . But then the actual strength is 500 / 501 or 0.998 . Hence, in this case Test A would judge (F) to be linked, when it is canonically convergent.

But perhaps an advocate of Test A will argue as follows, (B) and (F) are both canonically convergent such that when compared with argument (A), since arguments $(B)$ and $(F)$ are in the vast majority of circumstances convergent, either (B) or $(\mathrm{F})$ is sufficient to make it clear that there is a distinction to be made. At the same time, this does not mean that (B) and (F) are always convergent. In some circumstances, like the one sketched above, $(F)$ is linked despite the fact that in most circumstances it is convergent. Indeed, for many generally convergent arguments it may be possible to construct situations in which the argument is linked.

There are several problems with this proposal. Firstly, it assumes that in the majority of cases (B) and (F) are in fact convergent. But is it really true that on the vast majority of possible arrangements of Tom working and the mail coming, (F) turns out to be convergent? As we shall see below, there are plenty of possible circumstances such that (F), according to the Test, comes out linked. Secondly, it grants that even canonically convergent arguments are sometimes linked. But while it might be plausible to hold that certain problematic arguments are problematic precisely because in some circumstances these arguments are linked, but in others they are convergent, is it plausible to hold that canonically convergent arguments-arguments that are obviously or pre-theoretically convergent-can also be linked? Thirdly, the proposal requires a piecemeal acceptance of our intuitions concerning the linked/convergent distinction. Firstly, our intuitions concerning the difference between (A) and (B), or (A) and (F) are what motivate the linked/ convergent distinction in the first place. Secondly, finding out that in one circumstance the ordinary sum is greater than the actual probability, but that in another circumstance less, does not change our intuitions concerning whether $(B)$ or $(F)$ is linked or convergent, whether the arguer is offering two reasons or one. But if we accept Test A's results that (B) and (F) are sometimes linked and sometimes convergent, we need to give up the second intuition. But if our intuitions concern- 
ing (B) and (F) need to be given up, how can we trust our initial intuitions concerning the difference between (A) and (B), or (A) and (F)? But if we give up the first intuition as well, then the whole reason for the Test disappears. So the proposal requires us to keep the first intuition, but not the second, in order not to be self-defeating. But given that we accept the first intuition, what possible reason could be given for accepting the results of the Test over our second intuition, rather than the other way around?

The key problem for Test $\mathrm{A}$ is that it allows canonically convergent arguments, at least in some circumstances, to be linked. Assume for the moment that we are unwilling to accept this consequence. How might we modify Test $\mathrm{A}$ to avoid making our canonical arguments turn out to be both convergent and linked? Perhaps we should not interpret "jumps" as simply "greater than", but rather as "much greater than" or "significantly greater than".? For example, as Yanal points out, in a quite reasonable set of circumstances the ordinary sum of $(A)$ is 0.55 whereas the actual probability is 1 . The jump from 0.55 is clearly a significant jump, whereas the increase from 0.9711 to 0.998 apparently is not. Hence, an advocate of using ordinary summing might advocate:

Ordinary Summing Test B: For any argument with exactly two premises, the argument is linked if $p r_{1,2}$ is significantly greater than $o s_{1,2}$ and otherwise convergent.

Test B is the version of Yanal's test that Walton $(1996,181-82)$ advocates. $^{8}$ Clearly one significant question for advocates of Test $B$, is exactly how much greater is significant. Walton recognizes the importance of this question, but admits that no clear answer has been given (Walton, 1996, 135, 165-166).

Let us suppose for the moment that a universally uniform answer is possible. For example, perhaps, for all arguments, a jump is significant iff it is a jump of 0.25 or greater. Unfortunately, regardless of what fixed value is deemed the minimum to count as significant, Test B will either misclassify canonical arguments or classify one and the same canonical argument as linked in some circumstances and convergent in other circumstances.

Reconsider (F) yet again, but in the following circumstances. The mail comes only twice a week, on Wednesdays and Saturdays. Hence, $p r_{1}=0.5$. Tom goes to work on every Sunday, but only one day out of 100 for each weekday. So out of 100 weeks, Tom goes to work 105 days (100 Sundays, and one Monday, one Tuesday, etc ). Hence, $p r_{2}=5 / 105$ or approximately 0.0476 . The ordinary sum of $p r_{1}$ and $p r_{2}$ is approximately 0.5238 . But since the only day on which it is true that both the mail comes and Tom goes to work is Wednesday, $p r_{l, 2}=1$. But now compare (F) with (A). In (A) $o s_{1,2}=0.55$ and $p r_{l, 2}=1$. The jump of 0.45 in (A) was deemed significant and so Test B correctly judged (A) to be linked. But in the current situation with $(F)$ the actual probability jumps the ordinary sum by even more than 0.45 and so if the jump in (A) is significant, then so must the jump in (F) and so $(\mathrm{F})$ is once again judged to be linked. 
Now reconsider (A), but in the following circumstances. Cora, whose husband died not long ago, almost never goes into the study anymore because the study reminds her too much of her departed husband. Hence, given that she is in the study or in the kitchen, the probability that she is in the study is extremely low, whereas the probability that she is in the kitchen is extremely high. Suppose then that, given she is in either the study or the kitchen, the probability that she is in the kitchen is 0.99 . Continue to assume that the fact that she is not in the study, by itself, only makes it $10 \%$ likely she is in the kitchen. In this case $p r_{1}=0.99$ and $p r$, $=0.1$. Hence, $o s_{l, 2}=0.991$. But $p r_{l, 2}=1 .^{9}$

Now compare (A) and (F) again. The move to Test B from Test A was motivated by the claim that the jump from (F)'s ordinary sum of 0.9711 to $(\mathrm{F})$ 's actual probability of 0.998 , a jump of 0.0269 , is not a significant jump. But if a "jump" of 0.0269 is not significant, then a "jump" of 0.009 is certainly not, and according to Test B, (A), contra appearances, is convergent. Hence, either Test B misclassifies (A) in some cases or, despite (A)'s canonically linked appearance, $(A)$ is convergent in some cases.

Indeed, with enough ingenuity, circumstances can be made such that no matter what fixed value is deemed the minimum required for a significant jump, arguments such as (A) and (F) or (B) can be made to either both involve a significant jump or both not involve a significant jump. Either way, either Test B misclassifies at least one, if not both, of the canonical arguments or Test B allows at least one, if not both, of the canonical arguments to be in some circumstances linked and in some circumstances convergent. Hence, on the supposition that what counts as a significant jump is a fixed value, Test B fails to solve the problems of Test A.

Suppose, then, that an advocate of Test B admits that what counts as a "significant jump" can vary from one set of circumstances to the next. But what, other than a question-begging appeal to the very structure of the arguments in question themselves, could possibly justify the required variation so that (A) always turns out linked and (F) and (B) always turn out convergent? On what grounds could one argue that in the case of Cora being in her study only one time out of 100 , a jump of merely 0.009 is sufficient, but in the case of Tom working only one Saturday in 100, a jump of 0.0269 is not? In fact, what the extreme variability of the possible values of $p r_{1}, p r_{2}, o s_{l, 2}$ and $p r_{l, 2}$ for (A), (B), and (F) strongly indicates is that these values are largely independent of an argument's structure. If true, then only by appealing to this very structure could 'significant jump' be defined in order to give the intuitively correct answers. Indeed, if true, we most certainly should abandon appeal to the relative values of $o s_{t, 2}$ and $p r_{t, 2}$ to determine an argument's structure.

Perhaps then the problem is the definition of the ordinary sum itself. In the conclusion of his paper, Tyaglo claims, as William Grennan hinted, that the ordinary summing equations are "only approximations to accurate formulae" and that "correct formulae might be derived based on a relevant J.M Keynes' equation" 
(Tyaglo, 2003, 69). Let $k e_{1,2}$ stand for the result of Keynes' equation, in which case Keynes' equation is,

$$
k e_{1,2}=\operatorname{Pr}(\{\mathrm{C} \& \mathrm{P} 1\} \mid \mathrm{P} 2) /[\operatorname{Pr}(\{\mathrm{C} \& \mathrm{P} 1\} \mid \mathrm{P} 2)+\operatorname{Pr}(\{\sim \mathrm{C} \& \mathrm{P} 1\} \mid \mathrm{P} 2)]
$$

which Tyaglo calls "Keynes' formula for a two-premise convergent argument" (Tyaglo, 2003, 70). In other words, the result of Keynes' equation is just the probability, given $\mathrm{P} 2$, of both the conclusion and $\mathrm{P} 1$, divided by the sum of $(i)$ the probability, given $\mathrm{P} 2$, of the conclusion and $\mathrm{P} 1$ and (ii) the probability, given $\mathrm{P} 2$, of $\mathrm{P} 1$ and the negation of the conclusion.

Tyaglo's concern about using Keynes' equation is that "in a regular case this equation includes additional unknown variables and so isn't solvable" (Tyaglo, $2003,69)$. In other words, Keynes' ordinary summing equation is less likely to be solvable than Yanal's even if Yanal's is only an approximation. Indeed, one significant practical concern with the Ordinary Summing Test is that it requires being able to determine the conditional probabilities of $(i)$ the conclusion given each premise separately and (ii) the conclusion given both premises together. But in many cases these probability values are unknown and perhaps unknowable and merely guessing at the values is prone to yield erroneous results (See also Walton, 1996, 128).

Regardless of these practical concerns, the current issue is whether, even in the cases in which the relevant probabilities are known, either of these equations can be used to help determine whether an argument is linked or convergent. The arguments of this section and section 4 above show that the ordinary summing equations will not succeed. But if the ordinary summing equations are just approximations to Keynes' equation, then perhaps better results will be achieved if, instead of using $o s_{1,2}$ in the previous tests, we use $k e_{1,2}$. For example, reconsider (F) in the very first set of circumstances. Tom goes to work on 30 out of 100 Saturdays and 30 out of 100 Sundays and 88 out of 100 weekdays. Hence 440 of the 500 cases in which Tom goes to work, or $88 \%$, are cases in which it is both a weekday and the mail came. On only 30 of those 500 days, or $6 \%$, is it both the case that the mail came and it is not a weekday. Hence, $k e_{I .2}=0.88 /(0.88+0.06)$ or approximately 0.936 which is indeed equal to the actual conditional probability. So Keynes' equation, unlike the ordinary summing equation, generates the correct result for $(F)$.

But now reconsider (A). Given that she is not in the study, the probability that she is both either in the kitchen or the study and not in the kitchen is 0 -after all, it is impossible that she is either in the kitchen or the study and not in the study and not in the kitchen. But then $\left.k e_{12}=\operatorname{Pr}(\{\mathrm{C} \& \mathrm{P} 1\} \mid \mathrm{P} 2) /[\operatorname{Pr}(\{\mathrm{C} \& \mathrm{P} 1\} \mid \mathrm{P} 2)+0)\right]$ or just $\operatorname{Pr}(\{C \& P 1\} \mid P 2) / \operatorname{Pr}(\{C \& P 1\} \mid P 2)$ which is just 1 . Hence $k e_{1,2}=p r_{1,2}$ for (A) as well, and using $k e_{1,2}$ does not distinguish (A) from (F).

The real problem with Keynes' formula is that, contra Tyaglo, the equation is 
not Keynes' equation for convergent arguments, but rather his equation for calculating the probability of a conclusion given any two premises whatsoever (Keynes, $1962,149-150$ ). In other words, Keynes is providing a general formula for calculating $\mathrm{pr}_{l, 2}$ given any conclusion and any two premises. But if $k e_{1,2}$ just is $p r_{l, 2}$, then $k e_{12}$ will never differ from $p r_{l, 2}$ and we certainly cannot use $k e_{1,2}$ to demarcate linked from convergent arguments, because comparing the result of Keynes' formula with $p r_{l, 2}$ does not distinguish any arguments whatsoever.

\section{Conclusion}

While Walton advocates his own version of the Ordinary Summing Test, he admits that perhaps "we have not yet discovered the key counterexample that refutes the [Ordinary Summing Test] or shows it to be problematic" (Walton, 144). Whether any of the examples given here constitute by themselves a key counterexample, I do not know. But what all the examples together show is that no version of the Ordinary Summing Test successfully demarcates all canonical linked arguments from all canonical convergent arguments. Indeed, it appears that our judgements concerning the obvious cases are independent of the values of the conditional probabilities appealed to in the Tests. It is our intuitions that make us believe there is a distinction to be made in the first place and so given the choice between following the Test or sticking with our intuitions, we should stick with our intuitions. If the Ordinary Summing Test is unsuccessful, then no generalization of it will be successful either. ${ }^{10}$

\section{Appendix One}

Prove: If $p r_{1,2,3}=o s_{1,2,3}$ and none of $p r_{1}, p r_{2}$, and $p r_{3}$ is equal to 1, then the pattern is not any of $($ ii) $-($ iv).

Assume that none of the premises is alone sufficient for the conclusion, i.e., $p r_{1} \neq 1, p r_{2} \neq 1$ and $p r_{3} \neq 1$. Assume $p r_{1,2,3}=O s_{i, 2,3}$. Now suppose (iv) is the correct pattern.

(I) $o s_{l, 2,3}=o s_{l, 2}+\left(1-o s_{l, 2}\right) p r_{3}$. Since, by $\mathrm{C} 2$, the order of ordinary summing does not matter, $o s_{1,2,3}$ also equals $p r_{3}+\left(1-p r_{3}\right) o s_{1,2}$. [The equivalence of $o s_{1,2}+(1$ $\left.-o s_{l, 2}\right) p r_{3}$ and $p r_{3}+\left(1-p r_{3}\right) o s_{l, 2}$ can be quickly verified by some simple algebra.] Hence $p r_{l, 2,3}=p r_{3}+\left(1-p r_{3}\right) o s_{l, 2}$.

(II) Consider the two-premise argument whose first premise is just the conjunction of $\mathrm{P} 1$ and $\mathrm{P} 2$, viz.:

(X) $\mathrm{P} 1 \& \mathrm{P} 2, \mathrm{P} 3 / \mathrm{C}$.

The probability of $\mathrm{C}$ given both $\mathrm{P} 1 \& \mathrm{P} 2$ and $\mathrm{P} 3$ is the same as the probability of $\mathrm{C}$ given $\mathrm{P} 1, \mathrm{P} 2$ and $\mathrm{P} 3$, i.e., $p r_{1,2,3}$. Also, the probability of the conclusion given $\mathrm{P} 1 \& \mathrm{P} 2$ is exactly the same as the conditional probability of $\mathrm{C}$ given P1 and P2 as 
separate premises, viz., $p r_{1,2}$. The probability of the conclusion given $\mathrm{P} 3$ is just $p r_{3}$. Hence the ordinary sum of $(\mathrm{X})$ is equal to $p r_{1,2}+\left(1-p r_{1,2}\right) p r_{3}$. Again by $\mathrm{C} 2$ this is the same as $p r_{3}+\left(1-p r_{3}\right) p r_{l, 2}$. Since $(\mathrm{X})$ is convergent, $p r_{l, 2,3}$ is equal to the ordinary sum. Hence, $p r_{l, 2,3}=p r_{3}+\left(1-p r_{3}\right) p r_{l, 2}$.

(III) By the result in (I) $p r_{l, 2,3}=p r_{3}+\left(1-p r_{3}\right) o s_{l, 2}$. But by the result in (II), $p r_{l, 2,3}$ is also equal to $p r_{3}+\left(1-p r_{3}\right) p r_{1,2}$. Hence, by the transitivity of identity $p r_{3}+(1-$ $\left.p r_{3}\right) p r_{l, 2}=p r_{3}+\left(1-p r_{3}\right) o s_{l, 2}$. Subtract $p r_{3}$ from both sides. Hence, $\left(1-p r_{3}\right) p r_{l, 2}$ $=\left(1-p r_{3}\right) o s_{i, 2}$. Since $p r_{3}$ is at most 1 and at least 0 , and by supposition $\cdot 1,1-p r_{3}$ is not 0 . Hence, we can divide both sides by $1-p r_{3}$. Hence $p r_{l, 2}=o s_{t, 2}$. But since, in pattern (iv), $\mathrm{P} 1$ and $\mathrm{P} 2$ are linked, given the Ordinary Summing Test, $p r_{l, 2}>$ $o s_{l, 2}$. Hence, given our two initial assumptions, if the pattern is (iv) $p r_{l, 2}$ is both equal to and greater than $o s_{t, 2}$. This is impossible. Hence, the pattern cannot be (iv). By parallel reasoning the pattern cannot be (ii) or (iii). Hence, if $p r_{t, 2,3}=$ $o s_{1,2,3}$ and none of $p r_{1}, p r_{2}$, and $p r_{3}$ are equal to 1, then the pattern is not any of (ii)-(iv). QED

\section{Appendix Two}

The proof of this claim proceeds by mathematical induction.

Base Clause: If neither $p r_{1}=1$ nor $p r_{2}=1$, then $o s_{l, 2}<1$.

Suppose neither $p r_{1}=1$ nor $p r_{2}=1$, but $o s_{l, 2}=1$. Since $o s_{1,2}=p r_{1}+\left(1-p r_{1}\right)$ $p r_{2}, 1=p r_{1}+\left(1-p r_{1}\right) p r_{2}$. Subtract $p r_{1}$ from both sides. Hence $1-p r_{1}=\left(1-p r_{1}\right)$ $p r_{2}$. If we divide both sides by $1-p r_{1}$ we get the result that $p r_{2}=1$, which contradicts our initial assumption. Hence we cannot divide by $1-p r_{l}$, in which case $1-p r_{1}=0$, in which case, contra our initial assumption $p r_{1}=1$. Hence, if neither $p r_{l}=1$ nor $p r_{2}=1$, then $o s_{l, 2} \neq 1$. Since $o s_{l, 2}$ must be between 0 and $1, o s_{l, 2}$ $<1$.

Induction Clause: Provided that if for all $\mathrm{k}, p r_{k} \neq 1$, then $o s_{1, \ldots, k}<1$, then if for all $\mathrm{k}+1, p r_{k+1} \neq 1$, then $o s_{1, \ldots, k+1}<1$.

Suppose $(i)$ if for all $\mathrm{k}, p r_{k} \neq 1$, then $o s_{1, \ldots, k}<1$, and (ii) for all $\mathrm{k}+1, p r_{k+1} \neq 1$. Suppose, however, that $o s_{l, \ldots, k+1}=1$. Since $o s_{1, \ldots, k+1}=o s_{l, \ldots, k}+\left(1-o s_{l, \ldots, k}\right) p r_{k+l}$, then $1=o s_{l, \ldots, k}+\left(1-o s_{l, \ldots, k}\right) p r_{k+1}$. Hence, $1-o s_{l, \ldots, k}=\left(1-o s_{l, \ldots, k}\right) p r_{k+1}$. If we divide both sides by $1-o s_{l, \ldots, k}$, we get $p r_{k+1}=1$ which contradicts our supposition that for all $\mathrm{k}+1, p r_{k+1} \neq 1$. Hence, we cannot divide both sides by $1-o s_{l, \ldots, k}$, which means 1 $o s_{1, \ldots, k}$ must equal 0 . Hence, $1=o s_{l \ldots, k}$. But given that for all $\mathrm{k}, p r_{k} \neq 1$, and supposition (i), os $s_{1, \ldots, k}<1 . o s_{1, \ldots, k}$ cannot be both equal to and less than 1 . Hence, the supposition that $o s_{1, \ldots, k+1}=1$ must be wrong given suppositions $(i)$ and $(i i) . o s_{1, \ldots, k+1}$ must be between 1 and 0 and since it is not 1 , must be less than 1 . Hence, provided that $(i)$, then given that $(i i), o s_{1, \ldots, k+1}<1$. QED 


\section{Notes}

'Note that Yanal's 1991 (A) and (B) are not quite the same as his 2003 (A) and (B). I have opted for the latter because, unlike his 1991 examples, Yanal provides ordinary summing calculations for both his 2003 examples.

${ }^{2}$ Tyaglo uses the standard $\mathrm{p}(\mathrm{C} \mid \mathrm{P} 1, \mathrm{P} 2)$ and $\mathrm{p}(\mathrm{C} \mid \mathrm{P} 1, \mathrm{P} 2, \mathrm{P} 3)$ notation to articulate Yanal's formula and its generalizations. The problem is that $\mathrm{p}(\mathrm{C} \mid \mathrm{P} 1, \mathrm{P} 2)$ literally means the actual probability of the conclusion given the two premises, i.e., what I call $p r_{1,2}$, which, in most cases, is not the ordinary sum of $\mathrm{p}(\mathrm{C} \mid \mathrm{P} 1)$ and $\mathrm{p}(\mathrm{C} \mid \mathrm{P} 2)$. But the generalized ordinary sums are recursive on previous ordinary sums, not the previous actual probabilities.

${ }^{3}$ Vorobej (1995) also articulates a taxonomy involving convergent, linked, and hybrid arguments. However, other than Vorobej's "convergent" being the same as my "purely convergent" our taxonomies do not correspond.

${ }^{4}$ Given that the numbering of premises is irrelevant, patterns (ii) - (iv) are equivalent in the sense that any argument that is an example of one of those patterns, through a simple renumbering of the premises, can be made to be an example of either of the others.

${ }^{5}$ The probability assignments here assume that I am correct $75 \%$ of the time with respect to what I see relative to her being or not being in the kitchen. If we drop the relative to her being or not being in the kitchen, determining how the two premises interact is extremely difficult, if not impossible. For example, if I saw her heading for the bedroom and she's in the study, I am in one sense wrong about what I saw. But since seeing her head for the bedroom is also a case of seeing her not heading toward the kitchen and she is in fact not in the kitchen it is also a case in which I am right about what I saw.

${ }^{6} \mathrm{P}$ and $\mathrm{Q}$ are stochastically independent iff $\mathrm{pr}(\mathrm{P} \mid \mathrm{Q})=\operatorname{pr}(\mathrm{P})$. In other words, the fact that $\mathrm{Q}$ has no bearing on the probability of $P$ (and vice versa). The General Disjunction Rule is $\operatorname{pr}(P \vee Q)$ $=\operatorname{pr}(\mathrm{P})+\operatorname{pr}(\mathrm{Q})-\operatorname{pr}(\mathrm{P} \& \mathrm{Q})$. But by the Special Conjunction Rule, when $\mathrm{P}$ and $\mathrm{Q}$ are stochastically independent, $\operatorname{pr}(\mathrm{P} \& \mathrm{Q})=\operatorname{pr}(\mathrm{P}) \times \operatorname{pr}(\mathrm{Q})$ (Skyrms, 2000, 115, 121-122).

${ }^{7}$ Describing part of his earliest version of the distinction, Yanal writes "Reasons are DEPENDENT when together they make the overall strength of the argument MUCH GREATER than they would considered separately" $(1988,42)$. In Yanal 1991 and 2003, the "much greater than" locution disappears. Walton quotes Yanal's 1988 description, but ultimately adopts the locution "significantly greater" for his version of Yanal's test.

${ }^{8}$ Admittedly, Walton writes,

What is most important in judging an individual case is the structure of the argument, the indicator-words, and the evaluator's interpretation of how the argument is being used, in the context of dialogue, to make a point. In most cases, these three indicators are the main basis for making a judgement, and we may have no need at all to apply the Degree Supp. test. The test shouid be seen as more of an adjunct indicator that can be applied if these other indicators are not very decisive (181).

But the problem for the Degree Supp. test, i.e., the Ordinary Summing Test and its variants, is that it misclassifies canonical cases of linked and convergent arguments. But canonical cases are presumably canonical because they are unambiguous with respect to the primary evidence, i.e., with respect to their overt scheme, indicator words, and use. But if the Test misclassifies in the cases in which the primary evidence is unambiguous, why should we suddenly rely on the test to provide the correct answer when the primary evidence is ambiguous?

${ }^{9}$ Walton $(1996,129)$ also acknowledges the existence of these sorts of problematic cases for classifying (A).

${ }_{10}$ Ancestors of parts of this paper, most especially parts of section 4 and section 2, constitute parts of Goddu 2003, which is commentary on Yanal 2003 both of which were presented at the 2003 Informal Logic@25 Conference at Windsor, Ontario. 


\section{References}

Conway, David. (1991). "On the Distinction between Convergent and Linked Arguments," Informal Logic 13, pp. 145-158.

Goddu, G. C. (2003). "On 'Linked and Convergent Reasons-Again'." In Informal Logic at 25: Proceedings of the Windsor Conference, J. Anthony Blair, et al. (eds.), CD-ROM, Windsor, ON: OSSA, 2003.

Keynes, John Maynard. (1962). A Treatise on Probability. New York: Harper \& Row Publishers.

Skyrms, Brian. (2000). Choice and Chance. 4th ed. Belmont, CA: Wadsworth.

Tyaglo, Alexander V. (2002). "How to Improve the Convergent Argument Calculation," Informal Logic 22, pp. 61-71.

Vorobej, Mark. (1995). "Hybrid Arguments," Informal Logic 17, pp. 289-296.

Walton, Douglas. (1996). Argument Structure: A Pragmatic Theory. Toronto: University of Toronto Press.

Yanal, Robert J. (2003). "Linked and Convergent Reasons-Again." In Informal Logic at 25: Proceedings of the Windsor Conference, J. Anthony Blair, et al. (eds.), CDROM, Windsor, ON: OSSA, 2003.

Yanal, Robert J. (1991) "Dependent and Independent Reasons," Informal Logic 13, pp. 137-144.

Yanal, Robert J. (1988). Basic Logic. St. Paul: West Publishing Company.

G. C. Goddu

Department of Philosophy

University of Richmond, VA

U.S.A. 23173

ggoddu@richmond.edu 